\title{
El sistema defensivo del Antemural del Pacífico y Llave del Mar del Sur. Las fortificaciones de la Cuenca de Valdivia y la Bahía de Corral (Chile)
}

\section{Ester Prieto Ustio}

Universidad de Sevilla, Sevilla, España, esterprieto@hotmail.com

\begin{abstract}
From the Discovery of America, one of the priorities that had the Spanish Crown was to provide a good defensive system to all the territories to prevent for indigenous raids and try to curb piracy in the Caribbean area and Pacific surfaces.

Between the Sixteenth and Eighteenth Centuries in the American colonial space, fortifications were built following various models from the Old Continent, like proposals from Italy, France or Flanders.

One of the most sensitive areas was the Pacific and the key points for this defense were Valdivia Basin and Bay of Corral, and which were the Pacific and South Sea Key, a fortified system in which embody the great advances in architecture and engineering, and is a example of Spanish colonial military architecture.

We want to present the history of this important defensive system, its main enclaves, the people involved in its construction and operation during the Modern Age.
\end{abstract}

Keywords: Pacific, Fortress, Modern Age, Fortification.

\section{El Descubrimiento de América}

El Descubrimiento de América, realizado en la primera expedición colombina hacia las Indias en 1492, ha constituido uno de los episodios históricos más significativos de la humanidad. El devenir de los anales de Occidente adquirió un nuevo rumbo, y este acontecimiento, junto a la Caída de Constantinopla y la invención de la imprenta (ambos sucesos acaecidos en 1453) significaron el inicio de la Edad Moderna.

Esta etapa se caracteriza, a grandes rasgos, por el desarrollo de valores y conceptos como la comunicación, el progreso, la globalización y la razón, bases de la modernidad. La unión de territorios, hoy en día americanos, africanos y asiáticos supuso toda una revolución, creando incluso el término de "Era de los Descubrimientos" a estos siglos, ya que representaron la expansión de los dominios europeos, el intercambio cultural y social entre mundos totalmente diferentes y el acrecentamiento del comercio ultramarino

\subsection{Las fortificaciones en la América Española}

El Descubrimiento dio paso la Conquista española de los territorios americanos (principios y mediados del siglo XVI), y ésta, al dominio colonial mediante Virreinatos, responsabilidad de un "virrey", el encargado en gobernar y administrar una superficie en nombre de la corona en este caso, de España. Este gobierno se mantuvo desde el siglo XVI hasta principios del siglo XIX.

Desde el comienzo de la conquista en la zona de las Antillas, el Imperio español tuvo como 
prioridad dotar a esos nuevos asentamientos de un buen sistema defensivo primero, para prevenir ataques de los indígena y más adelante, para frenar la piratería que existía en todo el área caribeña y el Golfo de México, procedente por lo general de las Islas Británicas.

Una de las relaciones más importantes entre España y América fue el comercio entre ambos lugares, desde la plata, el oro, metales y piedras preciosas, madera, tintes, chocolate, café, patata... que se extraían de los nuevos territorios y se llevaban a la Península Ibérica, así como textiles, vino, aceite, víveres, libros, obras de arte... que se enviaban desde nuestra patria al Nuevo Mundo.

Este sistema comercial, denominado "Carrera de Indias", ha sido uno de los más fructíferos en la historia española, el cual estaba perfectamente organizado y controlado por instituciones creadas expresamente para ello, como la Casa de la Contratación (1503), el Consulado de Cargadores a Indias (1543), o el Almirantazgo (1624), ubicadas en Sevilla durante los siglos XVI y XVII , y trasladadas a Cádiz a partir de 1717.

Durante el siglo XVII, las principales amenazas de las zonas costeras y aguas pertenecientes a España dentro del marco americano provenían de la zona holandesa, que contaban con un amplio cuerpo de corsarios en diferentes acepciones: bucaneros, pechelinques, seadogs, hermanos de la costa, filibusteros...

En el siglo XVIII seguía existiendo el corsarismo, pero las fortificaciones y plazasfuerte tenían la función de impedir el paso a la coalición formada por Inglaterra y Holanda, los grandes enemigos de España en la época, que protagonizaron las llamadas "guerras de mar", unos enfrentamientos por el control de territorios europeos y colonias americanas entre estas dos naciones y la alianza hispano-franca.

Según el Diccionario de la Real Academia de la Lengua Española, una fortificación es "una obra, o conjunto de obras con que se fortifica un pueblo o un sitio cualquiera, construida con materiales duraderos para que sirva de defensa por tiempo limitado".
Ya en la Prehistoria nos encontramos con restos de estos reductos defensivos, como fueron los "talaiots" pétreos. Adquirieron un papel fundamental en el Imperio Romano (marcado por su carácter conquistador y el gran número de sus campañas de combate), el cual desarrolló los "castra" (campamentos militares), murallas y torres construidas con macizos bloques de piedra. En la Edad Media proliferarán un gran número de castillos, fortalezas, torres, urbes amuralladas... con la misión de defender cada feudo, señorío o reino. Seguirán usando la piedra, pero se incorporan materiales como el adobe, el ladrillo o la argamasa en este tipo de construcciones.

Una vez dentro de la Edad Moderna, estos sistemas evolucionaron notablemente a lo largo de los siglos, lo cual se puede observar en las fortificaciones americanas. En el siglo XVI, se siguen las directrices propuestas por la Escuela Italiana, que defiende el dotar de un sentido estético a estas obras (bajo principios clásicos); eliminar esa idea medieval de un fuerte cuadrado o poligonal sin articulación de por medio. Su gran novedad es la erección de fortalezas y castillos en zonas estratégicas para poder combinar y entrecruzar los fuegos $y$ contraataques entre estos edificios o el núcleo principal. El gran protagonista de esta escuela y período es Bautista Antonelli, el primer ingeniero militar llegado a las Indias, que se encargó de la construcción de las fortalezas de Los Tres Reyes del Morro y San Salvador de la Punta (La Habana, Cuba), el Fuerte de San Lorenzo (situado en la desembocadura del río Chagres, Panamá) e inició el sistema de murallas de Cartagena de Indias. Tenía un hermano, Juan Bautista Antonelli, también ingeniero militar, que trabajó en la zona mediterránea, como es el caso del Castillo de Santa Bárbara de Alicante y el Castillo de Benidorm.

Durante el siglo XVII, los grandes ingenieros procedían o seguían los propósitos de la Escuela Flamenca, que combinaba las técnicas holandesas y alemanas. Con un carácter muy orgánico, poseía una técnica muy cerebral, aplicando con gran rigor los principios matemáticos y geométricos, y un amplio conocimiento de los materiales constructivos. 
Comienzan a desarrollar el sistema de fortificación abaluartada, despiezando los lienzos y cortinas por medio de bastidores cuadrados o poligonales, pero todavía no se cultiva el baluarte en talud. Se eliminan las barbacanas de tradición medieval y se utilizan plenamente las impostas circulares.

Alguno de los representantes más insignes fueron Adrian Boot, que trabajó en Veracruz y Acapulco; Jaime Franck, encargado de remodelar San Juan de Ulúa o Enrico Martínez, que desarrolló su actividad profesional en Ciudad de México.

La Escuela Francesa, comenzó a formarse también en el siglo XVII bajo las ideas del Mariscal Vauban, pero tuvo su máximo desarrollo a partir del siglo XVIII, dotando de un método racional a todas las fortificaciones.

Como ya se hizo en el siglo anterior y enterrando el sistema del Medievo, la característica principal es el desglose de diversos núcleos y frentes fortificados, no sólo un único edificio. La gran aportación es el avance de un sistema abaluartado, creando así la "ciudadela", instalada dentro de una plaza y convirtiéndose en el último reducto defensivo (en el que refugiarse en caso extremo). La ciudadela se relaciona con la concepción del castillo medieval, pero ésta es más organizada que él anterior. Suscitó mucha polémica, ya que algunos autores señalan que desde esta parte se podía organizar la reconquista del resto del territorio si este fuera atacado, pero otros opinan lo contrario, puesto que el enemigo podría consolidarse llegando hacia ella.

Las defensas se erigen con proporciones monumentales, como es el caso de la Puerta de Tierra gaditana, y surgen elementos que complementan a la fortificación primordial, como baterías, medias lunas, hornabeques... También se añaden defensas exteriores a este conjunto para impedir la llegada del atacante, como fosos, caminos cubiertos, glacis, escarpas y contraescarpas... Aquí ya se utiliza la forma de talud y punto de diamante en las cortinas y baluartes, y se utilizan embrazaduras abiertas en su parte exterior en sustitución de los merlones, ya que éstas permitían el juego de los cañones para articular los fuegos cruzados.

\section{Las fortificaciones en la zona de la Cuenca de Valvidia y la Bahía de Corral (Chile)}

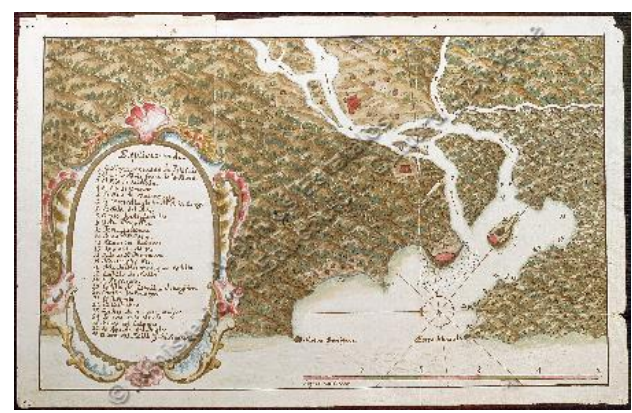

Fig.1- Plano de la ciudad de Valdivia (Archivo General del Indias, Sección de Mapas y Planos, 1785)

En el siglo XVIII, los Virreinatos de Nueva España y Perú eran dos importantes centros de riqueza de la Corona española en territorios americanos. El primero poseía dos vías de acceso: la Atlántica y la Pacífica; al segundo, más rico, gracias fundamentalmente a la explotación minera de la plata en el Potosí, sólo podía accederse a través de la costa Pacífica, vía Estrecho de Magallanes o Cabo de Hornos. Por ello ambos, fueron objetivo primordial de las potencias enemigas en la zona del Pacífico.

Valdivia fue fundada por Pedro de Valdivia (Archivo General de Indias, CHILE,18, R.1, N.1) en 1552, cien años antes de la construcción del sistema defensivo de la Bahía de Corral. Su economía se basaba en los lavaderos de oro del río Madre de Dios, permitiendo un rápido incremento de la población, ya que en 1570 vivían unas 1.200 personas aproximadamente, e incluso poseía siete iglesias. Sin embargo, en 1599, Valdivia fue destrozada por un ejército indígena, quedando abandonada durante 50 años.

En 1643, el navegante holandés Herckmans, arriba a las ruinas de Valdivia instalándose en ellas, pero de nuevo los indígenas lo expulsan ese mismo año. Esto atrae la atención del Virrey del Perú, el Marqués de Mancera, quien retomando la Real Cédula de 18 de mayo de 
1635, resolvió repoblar Valdivia y fortificar su puerto. El ingeniero mayor Constantino de Vasconcelos fue el encargado de la edificación de una serie de fortificaciones.

En 1645, esta primera avanzadilla, se instala en la isla de Constantino Pérez, iniciándose así la construcción del llamado Antemural del Pacífico y llave del mar del Sur, un sistema de fortificaciones destinado a proteger la refundación de Valdivia y el puerto de Corral, y que fue consagrado como Plaza militar de Valdivia, nombre que alterna, según las fuentes y las cédulas, con Plaza, fuerte y presidio de Valdivia.

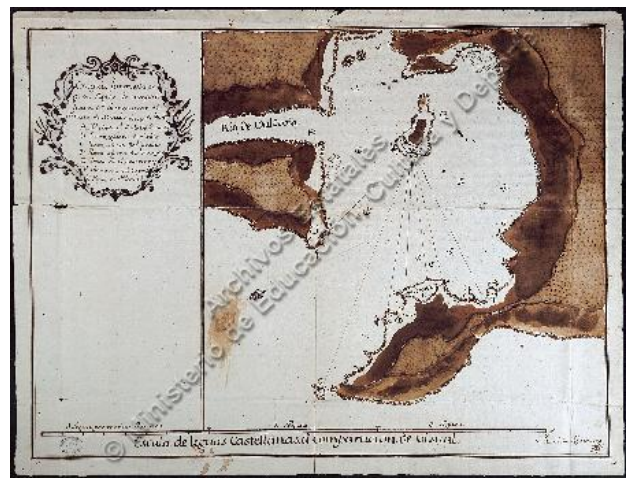

Fig.2- Croquis de la defensa de Valdivia (Archivo General de Indias. Sección de Mapas y Planos, 1744.

La ciudad es refundada con el rango de plaza fuerte y el Marqués de Mancera, la declara presidio, como una forma de asegurar los envíos de mano de obra forzada, necesaria para la construcción de los castillos. Las primeras construcciones consistieron en cuatro baterías protegidas por fajinadas o haces de ramas muy apretadas, ubicadas en Mancera, Corral, Amargos y Niebla. Entre los años 1650-1670, según el padre Rosales, tales asentamientos se transformaron en cuatro castillos sólidos con sus respectivos fosos y protegidos por los acantilados. Las potencias extranjeras (sobre todo Inglaterra) continuaran asediando la plaza, con las incursiones de Rogers (1708), Mareant (1720), Shelvocke y Clipperton (1725, 1721 y 1735), Lord Anson (1740) y Byron, todo ello con la intención última de provocar una ruptura en la zona del istmo, para dividir América y desarticular todo el mecanismo interno del Imperio Español.(Archivo Histórico Nacional DIVERSOS-COLECCIONES,27,N.41)

Durante todo el período colonial, el puerto de Valdivia llegó a ser considerado como una de las más importantes llave de entrada a los territorios de la Corona española en América, por eso se dotó con el más poderoso complejo defensivo de la costa occidental del continente, aunque Cartagena de Indias, La Habana y San Juan de Puerto Rico eran objetivamente los mejores centros defensivos.

Sin embargo, Valdivia no sólo debió estar preparada para enfrentarse a las potencias extranjeras, sino también para resistir los ataques de los indígenas, de ahí la construcción de un fuerte en el río Cruces, el Castillo San Luis del Alba de Cruces, para prevenir los ataques a la ciudad desde el continente.

Hacia el año 1670, se inicia una tercera etapa constructiva a cargo del Maestre de Campo Diego Joaquín de Martos, con el que se levantaron nuevas murallas y parapetos, y para el año 1681, el sistema contaba con 33 cañones distribuidos de la siguiente manera: 10 en el Castillo de San Pedro de Mancera, 2 en el Fuerte de San Francisco de Baldes, 5 en el castillo de San Luis de Alba, 8 en el Castillo de Niebla y 8 en la Plaza de Valdivia.

En 1762, una nueva Cédula Real, motivada por el ambiente hostil que se vivía entre las potencias marítimas europeas, decreta el traslado de la plaza de Valdivia a la isla de Mancera, para lo cual fue necesaria la construcción de nuevas habitaciones y almacenes.

Dos años más tarde, en 1764, comenzará una cuarta y última etapa de construcción dirigida por Juan Gardland y destinada, principalmente, a trabajos de mantención y consolidación. Previa a la llegada de Garland, el ingeniero José Birt levanta el fuerte de San Carlos, dos kilómetros al oeste de Amargos, y desmantela el fuerte de San Francisco de Baldes en 1748 por considerarlo ineficaz.

Para la óptima realización de sus obras de consolidación, Juan Garland instala numerosos hornos de ladrillo en la isla Valenzuela, actual 
isla Teja, y establece la manufactura de obras de carpintería en Mancera, junto con un hospital, cuarteles, un polvorín y almacenes. Entre los años 1780 y 1800 y conforme a su plan de perfeccionamiento de las defensas, levanta las baterías de la Aguada del Inglés y la del Barro en el margen sur del estuario, mientras que en la vertiente norte construye las de El Molino, la de Punta del Piojo y la de Carboneros. En 1787 se añadirá además, a este complejo entramado de fortificaciones, un camino real que lo unirá con el archipiélago de Chiloé; que ya por aquel entonces empezaba a cobrar importancia estratégica por encima de Valdivia, por ello se necesitaba un nexo de comunicación que permitiera trasladar refuerzos desde Valdivia a Chiloé.

Durante el transcurso de la Guerra de Independencia entre las colonias americanas y la Península, se redujo uno de los últimos enclaves españoles en Chile tras el desembarco en 1820 de Lord Cochrane.

\subsection{Funcionamiento del sistema defensivo}

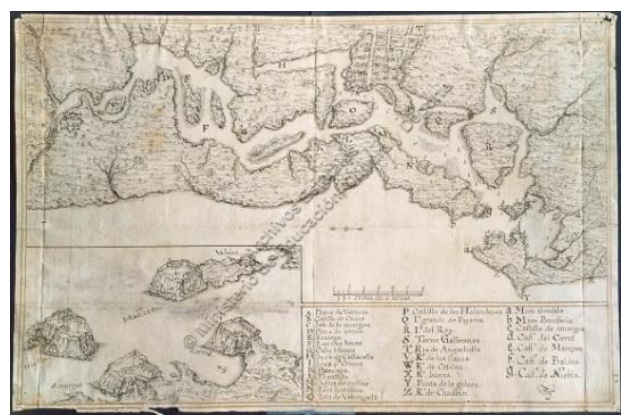

Fig.3- Vista general del sistema defensivo (Archivo General de Indias. Sección de Mapas y Planos, 1745)

Todo el sistema defensivo, el llamado Antemural del Pacifico y llave del Mar del Sur por los coetáneos, era un sistema perfectamente estudiado y calculado al milímetro para que funcionara como un todo, como un conjunto donde cada castillo, fuerte y batería cumpliera con una función específica ante cualquier posible ataque enemigo y defender así la plaza fuerte de Valdivia.
Su forma de operar era la que siguiente:

Ante el avistamiento de cualquier nave enemiga, el vigía del Morro Gonzalo avisaba con el cañón que poseía para tal fin, y de esa forma se alertaba a la artillería del resto del conjunto de la bahía, consiguiendo así tiempo suficiente para ocupar posiciones y encender los hornillos donde se preparaba la bala roja. Cuando las naves se avistaban en la costa, el Gobernador era el responsable de permitir la entrada de ésta en la bahía u ordenar su rechazo.

En el hipotético caso de que el barco fuera enemigo, se producía el combate. El navío intentaría por cualquier medio arribar a alguna de las playas existentes en la bahía para desembarcar. Si navegaba por la costa occidental, el barco debía esquivar a estribor los fuegos sucesivos de San Carlos, El Barro y Amargos, las baterías alta y baja de Chorocamayo y recibir a babor los disparos de Niebla. La artillería usaba aquí las piezas de mayor calibre, e intentaba utilizar los elementos más pequeños y ligeros para mitigar la falta de movilidad de su artillería pesada. Sus disparos eran balas encadenadas, $\mathrm{o}$ las llamadas "palanquetas", para conseguir romper el velamen de la nave y así reducir las maniobras de evasión de ésta. Una vez conseguido este objetivo, se podía asegurar el tiro ante la falta de movilidad del enemigo y utilizar la bala roja para dar fuego al navío.

$\mathrm{Si}$ el barco pasaba "limpio" estas primeras andanadas y llegaba al surgidero de Corral, se encontraría con toda su artillería, más las baterías de Chorocamayo, alta y baja. Si ante semejante fuego se intentara desembarcar tropa en la playa, entraría en acción la batería de El Bolsón. Para llegar a la plaza fuerte de Valdivia, las embarcaciones tenían que entrar necesariamente por los ríos, encontrándose entonces con la artillería de Mancera, Piojos y Carboneros.

Otra táctica enemiga que podría ser empleada era la del desembarco directo en las playas más cercanas al mar. Una vez en tierra, era factible tomar los castillos y baterías uno a uno desactivando así toda la defensa para permitir la entrada de navíos en el fondeadero. 
Para evitar estos desembarcos, las playas no poseían acceso desde tierra, teniendo que acceder los soldados desde el mar, debido a la falta de caminos para poder acceder a los castillos, que junto con la abundancia de vegetación en la zona, las rocas y lo agreste del terreno en definitiva, impedirían la invasión. Para ayudar a este propósito también había orden, en caso de ataque, de destruir los puentes que hubiera por el camino.

$\mathrm{Si}$ las tropas enemigas conseguían burlar estas precauciones en su desembarco, tenían que navegar por el río Valdivia para hacerse con la ciudad. El conjunto defensivo ejercía así un poder disuasorio ante cualquier eventual ataque.

Realmente esta era la verdadera arma de la ciudad, ya que en muchas ocasiones, el sistema se encontraba desabastecido de materiales, con tropa insuficiente o inexperta en artillería, además las humedades del terreno hacían que la mayor parte de las veces la pólvora se encontrase en mal estado.

\subsection{Principales enclaves del Antemural del Pacífico y Llave del Mar del Sur}

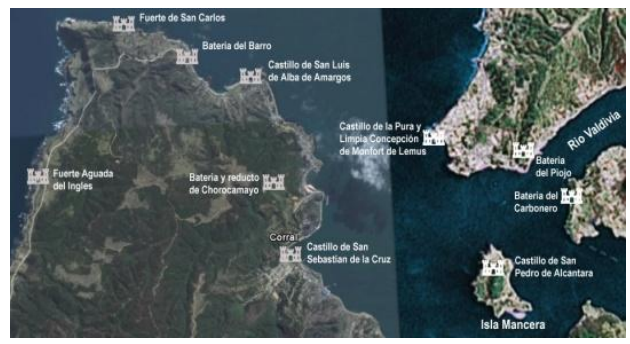

Fig.4- Situación actual de las zonas defensivas de la Cuenca de Valdivia y Bahía de Corral (FSMLR, 2013)

El conjunto de fortificaciones de la Cuenca de Valdivia y Bahía de Corral es una creación de gran mérito, ya que en ella se plasman los grandes avances y valores de la ingeniería y arquitectura de la Edad Moderna, siendo además uno de los mejores exponentes de la arquitectura militar colonial española en el continente americano.

$\mathrm{Su}$ aprovechamiento de las condiciones del terreno y de los materiales disponibles en su entorno, así como la ingeniería militar asociada a una táctica defensiva extraordinariamente concebida, conforman un escenario único para comprender la época de disputa de las naciones europeas por las riquezas americanas.

Los principales puntos de este sistema defensivo eran los siguientes:

- Castillo y Plaza Colonial de la Isla Marqués de Mancera

Formado por diferentes elementos como la Casa del Castellano, la Iglesia San Antonio, la Casa del Capellán, el Cuerpo de Guardia, la bóveda de zaguán, depósito de pólvora, calabozo, los baluartes, los Fosos Este y Oeste, las Cortinas Este y Oeste, baterías, hospital, aserraderos...

Durante el siglo XVIII, la isla alcanzó un alto valor estratégico, cuyo origen encontramos en el proyecto del Gobernador Pedro Moreno y Pérez (1731), de trasladar allí la plaza de Valdivia, lo cual se implementará bajo el gobierno Manuel de Amat y Junient. (1753-1767). (Archivo General de Indias CHILE,440)

Aunque en un primer momento se multiplicaron las construcciones y su población, lo cierto es que con el tiempo va a perder relevancia debido al ínfimo papel que representará en el conjunto del sistema defensivo. El progreso de las técnicas navales (que determinarán la construcción de nuevos fuertes y baterías) influirá poderosamente en la inhibición de su valor estratégico.

- Castillo de la Pura y Limpia Concepción de Monfort de Lumus de Niebla "Fuerte de Niebla"

Se trata de uno de los espacios con mayor valor patrimonial, ya que constituía la puerta de entrada al sistema desde Valdivia, posee una gran variedad de estilos castrenses y se encuentra muy bien integrado con el paisaje valdiviano.

Originalmente, el Castillo de Niebla contó con catorce cañones y murallas de piedra de altura poco considerable. Este último hecho determinó que el plan de remodelación y reforzamiento de la fortaleza, puesto en ejecución a fines del siglo XVIII, contemplara su refacción. Su muro fue ampliado, elevado y reparado, y se agrandaron 
algunas de sus dependencias internas; además, se excavó un foso en su alrededor. Contaba con una batería con 18 cañones de 24 libras y bala roja, horno para las balas, polvorín, capilla... Su guarnición era de aproximadamente 200 hombres. Entre 1715 y 1718 se monta una batería capaz de 16 cañones y se construyen dos almacenes, un Cuartel para la Infantería, la Casa del Castellano y una iglesia.

- Castillo de San Sebastián de la Cruz de Corral. Fuerte De Corral.

Compuesto por tres elementos unidos todos en el año 1767: Castillo de San Sebastián de la Cruz, más al sur, que data de 1645 , proyectado por Juan Garland; Batería de la Argolla, más al norte, que data del año 1764; y al centro un gran muro que unificó el Castillo, La Cortina (1767). Poseía un foso de 40 varas de ancho por 8 de de profundidad, cuarteles de piedra con capacidad para 200 hombres y sus muros contaban con 21 cañones todos provistos de bala roja. En 1683 es defendido por 34 hombres, 4 artilleros y 1 condestable, necesitándose más de 80 hombres de guarnición y por lo menos un artillero por pieza; en noviembre de 1686 contaba con un almacén de pólvora. La fortaleza no sufre mayores variaciones respecto del siglo XVIII. En1748 tiene un cuerpo de guardia de piedra con dos divisiones, una iglesia, la Casa del Gobernador, la del Castellano, el cuartel y una muralla que rodea las construcciones, cuyo estado era malo debido a la crudeza del clima. Hacia 1770, se levantan dos nuevas baterías denominadas del Bolsón y Argolla, ambas se unen a la muralla, dando al castillo la forma que actualmente conocemos

- Castillo de San Luis de Alba de Amargos. Fuerte de Amargos.

En su origen, se componía de una planchada de seis piezas de artillería y foso de más de media pica de alto. Su denominación se debe a que en el lugar donde se ubica existían varios manzanos, cuyo fruto no era precisamente dulce.

Rehecho por el Gobernador Diego de Martos, a principios de 1677, se le construye un Baluarte al frente del morro que mira hacia el mar, el cual cuenta con 32 pies geométricos, todo de piedra firme, sobre la cual se hizo una explanada, capaz de contar en ella doce piezas gruesas, cuando entes no cabían más de cinco, la obra estuvo a cargo de Buitrón y Mujica, la cual requirió más de 100.000 piedras, y a la cual también se le agregó un muelle de 30 varas en el mar, más a una capilla dedicada a la Virgen del Pilar. Durante las últimas décadas del siglo XVIII, el castillo será sometido a reparaciones, reedificándose una de las baterías que dan al mar, estrechándose el cuartel de la tropa y arreglándose sus muros.

- Fuerte de San Carlos

El fuerte de San Carlos está situado en la punta rocosa del morro, en la entrada del río Valdivia. Está compuesto por el fuerte propiamente dicho en la parte continental y la batería que se encontraba en un islote frente a él. Construido por Antonio Birt hacia 1763, se realizaron modificaciones hacia 1780, contaba con 6 cañones y un hornillo de bala roja. Como guarnición aproximada contaba con 100 hombres y era punto estratégico para defender la entrada a la Bahía de Amargos.

\section{Conclusiones}

Como hemos podido comprobar en este breve estudio, los sistemas construidos alrededor de los siglos XVII y XVIII para la defensa del Antemural del Pacífico y Llave del Mar del Sur, situados concretamente en la cuenca del río Valdivia y la Bahía de Corral, fueron una obra cumbre de la ingeniería militar hispana en territorios americanos, permitiendo defender todas las posesiones y riquezas del imperio español. En estas fortificaciones se reflejan todas las innovaciones desarrolladas en Europa, que rápidamente serán incorporadas en el Nuevo Mundo durante diferentes etapas constructivas en las que la finalidad era la defensa y el bloqueo de la entrada del enemigo. 


\section{Referencias}

Angulo, S. E. (1997), "La Artillería y los Artilleros en Chile. Valdivia y Chiloé como antemural del Pacífico" in Militaria: revista de cultura militar", 10, pp. 237-264

Amores, F.; Domínguez, E. L, (2008), Las Fortificaciones y El Mar, Ayuntamiento de Alcalá de Guadaira, Alcalá de Guadaira.

Calderón Quijano, J.A, (1996), "Fortificaciones Españolas” en América y Filipinas, Ed. Mapfre, Madrid.

Calderón Quijano, J. A.(1990), "Ciudades Costeras Españolas e Hispanoamericanas", in Actas de las VII Jornadas de Andalucía y América Sevilla, Ed. Universidad de Santa María de la Rábida.

Calderón Quijano, J.A, (1988), "Visión General De Las Fortificaciones Indianas en Los Distintos Frentes Continentales", in II Congreso de Historia Militar, Zaragoza, pp.150-155.

Fundación Santa María La Real (2013), Plan de manejo sobre la gestión cultural de la zona de Valdivia y Corral, Aguilar de Campoó, sin publicar.

Gutiérrez, R. (2000), Fortificaciones En Iberoamérica, Ed. El Viso, Madrid.

Tribaldos, L., Viforcos, M. I. (2009), Historia General De Las Continuadas Guerras y Difícil Conquista Del Gran Reino y Provincias De Chile, Desde Su Primer Descubrimiento Por La Nación Española, En El Orbe Antártico, Hasta La Era Presente, Ed. Universidad de León, León.

Urbina, M. X, (2009), La Frontera De Arriba En Chile Colonial: Interacción Hispano-Indígena En El Territorio Entre Valdivia y Chiloé e Imaginario De Sus Bordes Geográficos, 1600 -1800, Ed. Universitarias de Valparaíso, Valparaíso.

Villalobos R.S., (1991), Historia De Chile, Editorial Universitaria, Santiago de Chile.

V.V.A.A, (2001), Actas II Jornadas Sobre Fortificaciones Modernas y Contemporáneas : Mediterráneo Occidental (1500-1936), Ed. Áglaya, Cartagena. 